\title{
Dispersing Effect of Core Cross-linked Star Polycarboxylate Superplasticizers on the Cement Paste
}

\author{
Jinlong Zhang ${ }^{* 1,2}$, Furen Huang ${ }^{1}$, Xiaolong Chen ${ }^{1}$, Kaihong Zhong*1,2 and Haijun $\mathrm{Xu}^{1}$ \\ ${ }^{1}$ Guangzhou Institute of Building Science Co., Ltd., Guangzhou, People's Republic of China \\ ${ }^{2}$ Guangzhou Municipal Construction Group Co., Ltd., Guangzhou, People's Republic of China
}

\begin{abstract}
Researches on topological modification of polycarboxylate superplasticizers to improve the water-reducing or slump-retainning properties have been a hot topic in rencent years. Herein, a core crosslinked star polycarboxylate superplasticizers (SPCE) were synthesized by crosslinking the comb polycarboxylate superplasticizers (CPCE) in aqueous solution. Crosslinkers and spacing monomers were added into the polymerization to promote the formation of hydrophobic core. A larger molecular weight with $75 \%$ of yields of the SPCE were observed by gel permeation chromatography (GPC). The dispersing effect of SPCE on the cement paste was then investigated by varying the dosage of crosslinkers and spacing monomers. It was found that the dispersing ability of SPCE became weakened compared to the CPCE. More of the crosslinkers led to smaller flow spread, as revealed by cement paste experiments. Introduction of the spacing monomers contributed to the formation of SPCE. However, the dispersing ability was also weakened. Best conversion efficiency and water-reducing ability was obtained when the molar ratio of crosslinker and spacing monomer to CPCE was set as 3:5:1.
\end{abstract}

\section{Introduction}

Polycarboxylate superplasticizers (PCE), which featured by the properties of low dosage, high water-reducing, and excellent slump-retainning et al, have been proved to be indispensible materials in concrete. ${ }^{[1,2]}$ The steric hindrance of the long side chains and the electrostatic repulsion of carboxyl groups of PAA made PCE a better admixtures in concrete than lignosulfonate and naphthalene typed superplasticizers.

However, factors such as the shortage of natural sands, quality fluctuation of the concrete original materials and long-distance delivery, have put forward much higher request on the adaptability and slumpretainning ability of PCE. Traditional PCE with comb structure has exhibited its limitation on adjusting these new requirements.

As one kind of the polymers, the structure or the functionality of PCE could be well-designed by changing the polymerization methods or adding extra monomers. So in recent years, lots of researches on PCE modification, such as crosslinked, multiple-armed or spherical PCE have been reported. ${ }^{[1,2]}$ In our previous works, a nano-PCE with outstanding slump-retaining ability was built by adding the styrene as the hydrophobic segments. The ester group was encapsulated in a hydrophobic core, and hydrolyzed gradually under the alkaline environment of cement. As a result, the flow of cement paste could be maintained for $4 \mathrm{~h}$ with only $13.7 \%$ loss, and the hydration of cement could be effectively delayed. ${ }^{[5]}$
Star-shaped PCE (SPCE), which displayed a spherical structure is reported to have low viscous, multifunctional properties. ${ }^{[6,7]}$ Traditional SPCE was built with "core-first" method by constructing a crosslinked core. For instance, Liao et al reported a "four-arms" star polycarboxylate superplasticizers esterification-copolymerization. The star PCE exhibited good paste dispersion and fluidity retention. ${ }^{[8]}$ However, in the process of synthesis, a hydrophobic crosslinking core should be synthesized first in organic solvent $\mathrm{CH}_{2} \mathrm{Cl}_{2}$, which is unsuitable for massive application.

In this manuscript, a novel SPCE was synthesized by crosslinking the core with "arm-first" reversible addition-fragmentation chain transfer (RAFT) polymerization in aqueous media. ${ }^{[9,10]}$ Crosslinkers and spacing monomers were introduced to promote the formation of SPCE. The structure was clearly characterized, and the effect of the crosslinking density and spacing monomers on the water-reducing ability of SPCE was also investigated.

\section{Materials and methods}

\subsection{Materials}

All the chemical agents, such as 4-cyanopentanoicacid dithiobenzoate (CPADB), poly (ethylene glycol) methyl ether methacrylate (MPEG, $\mathrm{Mn}=950 \mathrm{~g} / \mathrm{mol}$ ), methacrylic acid (MAA), 4,4'-Azobis (4-cyanovaleric acid) (ACVA), ethylene glycol dimethacrylate (EGD) and butyl

\footnotetext{
* Corresponding author: zhangi1117@126.com;

42325231@qq.com.
} 
methacrylate (BMA), were purchased from Aladdin Industrial Co.Ltd. (Shanghai, China) and purified before used. The acetate buffer solution $(\mathrm{pH}=6.0)$ was prepared by mixing $2.86 \mathrm{~g}$ acetic acid with $78.09 \mathrm{~g}$ sodium acetate in $1000 \mathrm{ml}$. Standard Portland cement (P.I 42.5, CUCC, Chinese standard GB 8076-2008) was purchased from Shandong Lucheng Cement Co. Ltd., China.

\subsection{Synthesis of CPCE}

The CPCE was synthesized via RAFT-mediated aqueous polymerization. A mixture of CPADB, MPEG, MAA, ACVA were added into the buffer solution, where the molar ratio was set as CPADB:MPEG: MAA: $\mathrm{ACVA}=$ 1:60:20:0.25. The mixture was degassed with nitrogen at $0^{\circ} \mathrm{C}$ for $0.5 \mathrm{~h}$ before immersed into an oil bath at $70^{\circ} \mathrm{C}$. The polymerization was then kept for $6 \mathrm{~h}$ and quenched in an ice bath. The solution was further dissolved in tetrahydrofuran, followed by precipitating in n-hexane for three times. The CPCE was obtained by dried in vacuum overnight to get rid of the solvent.

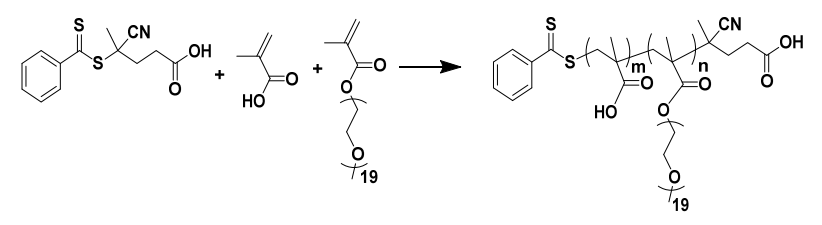

Scheme 1. Synthesis route of CPCE

\subsection{Synthesis of SPCE}

The SPCE was synthesized via a RAFT-mediated polymerization by core crosslinking. CPCE, EGD, BMA and ACVA were added into the deionized water. The mixture was degassed with nitrogen at $0^{\circ} \mathrm{Cfor} 0.5 \mathrm{~h}$ before immersed into an oil bath at $65^{\circ} \mathrm{C}$. The polymerization was kept for $6 \mathrm{~h}$ and quenched in an ice bath. A homogeneous solution was then obtained by dialyzed in deionized water for 3 days. The mass concentration of SPCE was finally confirmed by being dried in the hot oven at $105^{\circ} \mathrm{C}$ for $4 \mathrm{~h}$.

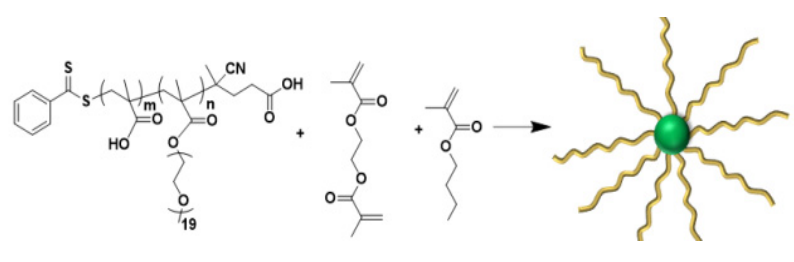

Scheme 2. Synthesis route of SPCE

\subsection{Characterizations}

${ }^{1} \mathrm{H}$ NMR spectra were recorded on a Bruker $400 \mathrm{MHz}$ AVANCE III spectrometer. The internal solvent proton signals at $\delta=4.75 \mathrm{ppm}$ of $\mathrm{D}_{2} \mathrm{O}$ was used as the reference. FT-IR spectra was measured on a BrukerVertex 70 spectrometer, each spectrum was recorded between $400 \mathrm{~cm}^{-1}$ and $4000 \mathrm{~cm}^{-1}$. The number average molecular weights $(\mathrm{Mn})$ and polydispersity indices (PDIs) of polymers were measured by a gel permeation chromatography (GPC) apparatus with $0.1 \mathrm{M} \mathrm{NaNO} \mathrm{Na}_{3}$ as the eluent.

\subsection{Dispersing test in cement paste}

Cement paste test was performed according to the procedure of the National Standard of China GB/T 80772012 , the mass concentration of SPCE was set as $8 \%$, and the water/cement ratio by weight was fixed at 0.30 . the procedures were as follows: $300.00 \mathrm{~g}$ standard cement, deionized water, and SPCE was was slowly agitated for $2 \mathrm{~min}$, followed by $15 \mathrm{~s}$ suspension and quick agitation for $2 \mathrm{~min}$. The Paste was then poured into a mini cone, which was placed on a transparent glass plate and then vertically lifted to make the paste flow. The diameter of the cement paste was then measured twice at two directions perpendicular to each other after 30 s for spreading.

\section{Results and discussions}

\subsection{Synthesis and characterization of SPCE}

The "arm-first" approach was used to synthesize the core-crosslinked star polycarboxylate superplasticizers. The comb polycarboxylate superplasticizers (CPCE) was first synthesized by RAFT-mediated aqueous polymerization with the molar ratio of acid to esters was set as 3:1. In the acetate buffer solution $(\mathrm{pH}=6.0)$, the CPADB acting as the chain transfer, could be totally soluable, which ensured that the polymerizaiton could be carried on in aqueous media. The CPCE was then used as the chain transfer agents to trigger the crosslinking reaction to give SPCE (Fig.1). During the crosslinking process, the linkers EGD caught CPCEs to form a hydrophobic core, and the BMA being as the spacing monomers were used to reduce the rigidity of EGD.

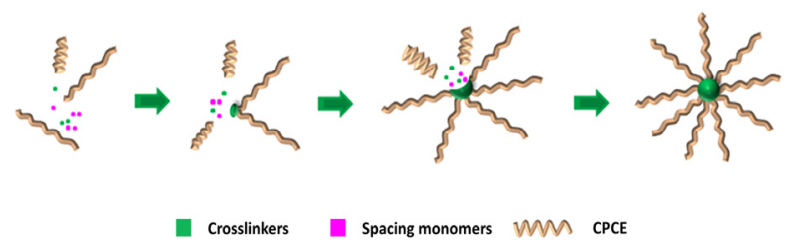

Fig. 1. The formation mechanism of SPCE

The chemical structure of SPCE was characterized by ${ }^{1} \mathrm{HNMR}$ as shown in Fig.2. Same with the spectra of CPCE, the peaks at $4.0 \mathrm{ppm}, 3.5 \mathrm{ppm}$ and $3.2 \mathrm{ppm}$ belongs to hydrogen proton of $-\mathrm{COOCH}_{2} \mathrm{CH}_{2-}$, $-\mathrm{OCH}_{2} \mathrm{CH}_{2}$ - and $\mathrm{CH}_{3} \mathrm{O}$ - in MPEG segments, respectively. The broad peak of 1.0-1.7 ppm belongs to the main chains. Hydrogen protons of the crosslinkers EGD and spacing monomers BMA could not be detected because of the high hydrophobicity in the core.

FT-IR was also performed to analyze the structure of SPCE. Due to the same groups in CPCE and SPCE, the spectra of SPCE was not so differernt from CPCE. According to Fig.3, the absorption peak at $1724 \mathrm{~cm}^{-1}$ 
belongs to the $-\mathrm{C}=\mathrm{O}$ in MAA 、 $\mathrm{MPEG} 、 \mathrm{EGD}$ and BMA, seperately. Peaks at $1100 \mathrm{~cm}^{-1}$ and $1354 \mathrm{~cm}^{-1}$ belongs to vibration of the $-\mathrm{C}-\mathrm{O}-\mathrm{C}-$ and $-\mathrm{OCH}_{3}$ in MPEG segments, the stretching vibration absorption peak at $2930 \mathrm{~cm}^{-1}$ belongs to the $-\mathrm{CH}_{2}-\mathrm{CH}_{2}$ - in the backbone.

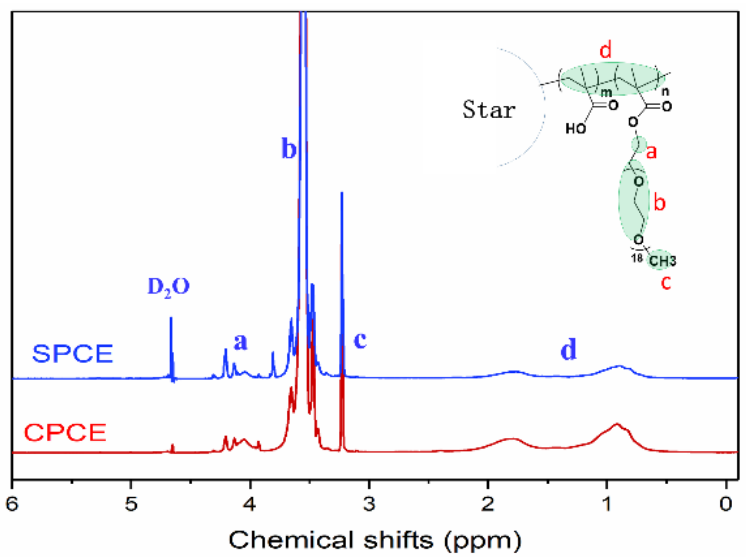

Fig. 2. The ${ }^{1} \mathrm{HNMR}$ spectra of $\mathrm{CPCE}$ and SPCE

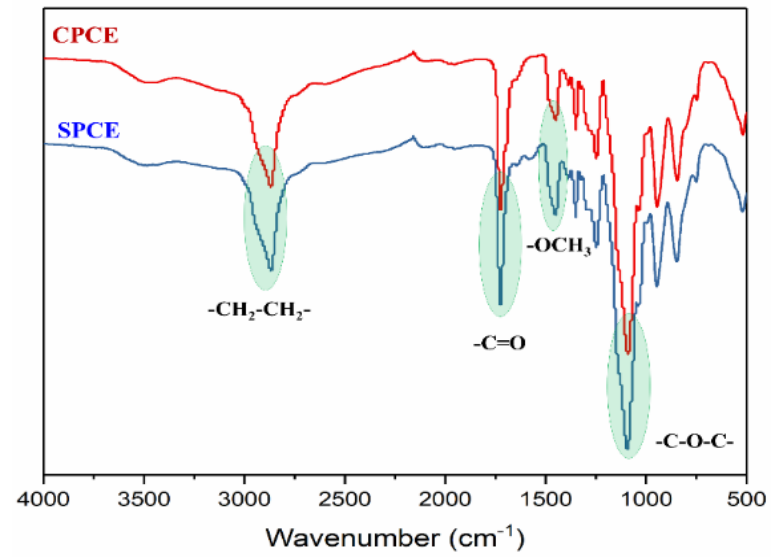

Fig. 3. The FT-IR spectra of CPCE and SPCE

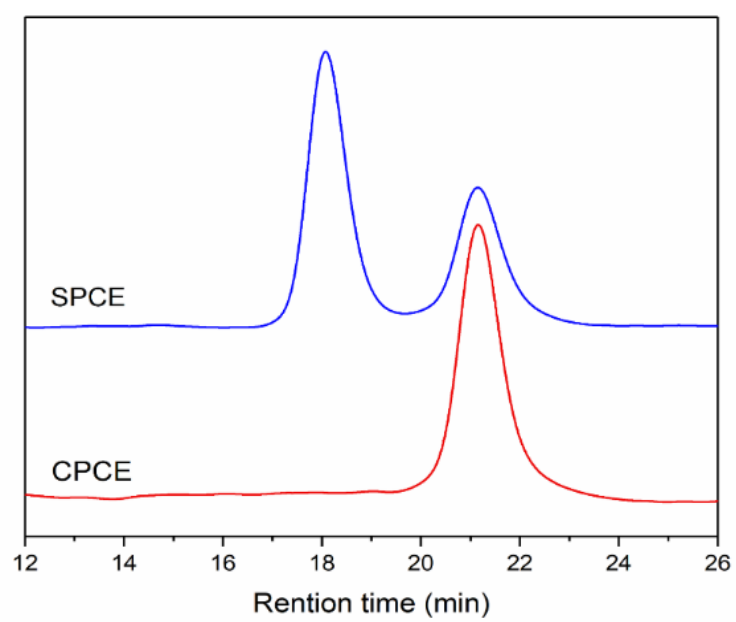

Fig. 4. The GPC spectra of CPCE and SPCE

In order to confirm the formation of SPCE, molecular weight and dispersity index were characterized by gel permeation chromatography (GPC). As shown in Fig.4, a spectrum with two independent peaks was obtained. The peak at the retention time of $21 \mathrm{~min}$ belongs to the signal of the non-crosslinked CPCE. A bigger peak that appeared much earlier than CPCE was obtained, which means that a larger molecular weight of polymers was got by core crosslinking. The yield of SPCE was then calculated to be almost $75 \%$ by counting the integral area of two peaks. The results of ${ }^{1} \mathrm{HNMR}$, FT-IR and GPC demonstrated that the SPCE was successfully obtained.

\subsection{Dispersing ability of SPCE}

The dispersing ability of PCE comes from the steric and electrostatistic repulsion provided by the long chains and negative carboxyl groups. As the SPCE exhibited a much more complicated topological structure than CPCE, it should display a better water-reducing properties. Herein, the effect of crosslinkers and spacing monomers on the dispersing ability of SPCE was clearly investigated.

\subsubsection{Effect of crosslinking density}

Effect of crosslinking density on the dispersing ability of SPCE was investigated by varing the dosage of crosslinkers. The molar ratio of corsslinkers to CPCE was set as $3: 1,5: 1,8: 1,10: 1,12: 1,15: 1$, separately. The cement paste flow test was then performed with addition of SCPE at solid dosages of $0.16 \%$ by weight of cement (bwoc). As shown in Fig.5, with the crosslinkers changing from 3 to 15 , the fluidity decreased from $275 \mathrm{~mm}$ to almost $100 \mathrm{~mm}$. A situation must be noted that at the same solid dosage of $0.16 \%$, the CPCE was overmixed, which means the CPCE showed a better dispersion effect.

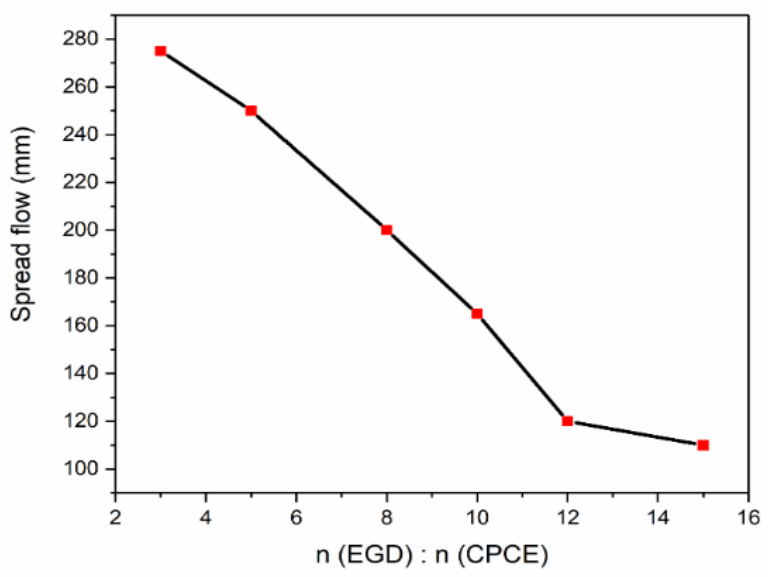

Fig. 5. Spread flow of cement paste under different crosslinking density

The reason was speculated as follows: the SPCE was featured with a 3D sphere structure, therefore, when the SPCE was added into the cement paste, only small proportion of the arms could act on the cement particles, the rest would be left useless in the paste. The more crosslinkers used, the more arms were bounded in one SPCE molecules, which means there would be more arms could not exert their functions. As for the CPCE, 
simple structure makes sure that all the chains could act on the cement particles, so the water-reducing ability of CPCE was much better than SPCE. The prime molar ratio of crosslinkers to CPCE was chosen as 3:1.

\subsubsection{Effect of spacing monomers}

As aforementioned, the introduction of spacing monomers BMA into the polymerization could decrease the rigidity of hydrophobic core provided by crosslinking, thus promote the formation of SPCE. In order to confirm the effects of BMA, the GPC and cement paste flow test were performed.

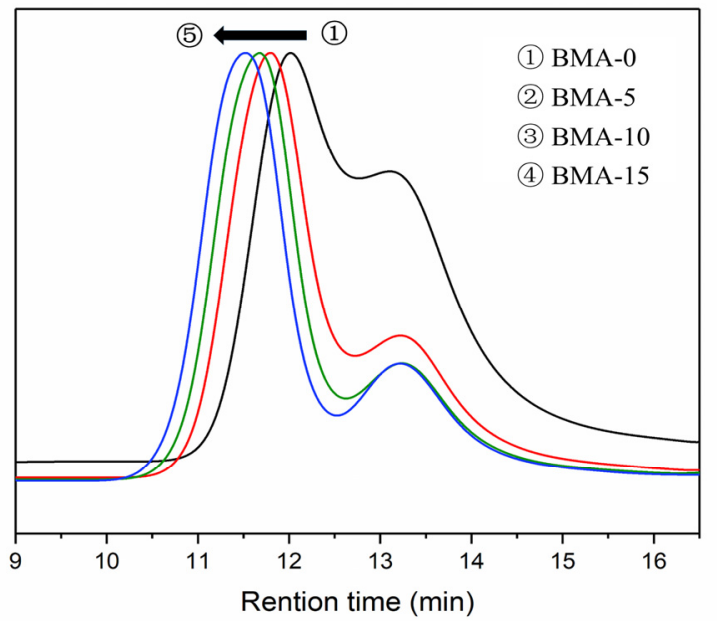

Fig. 6. Effect of BMA on the formation of SPCE

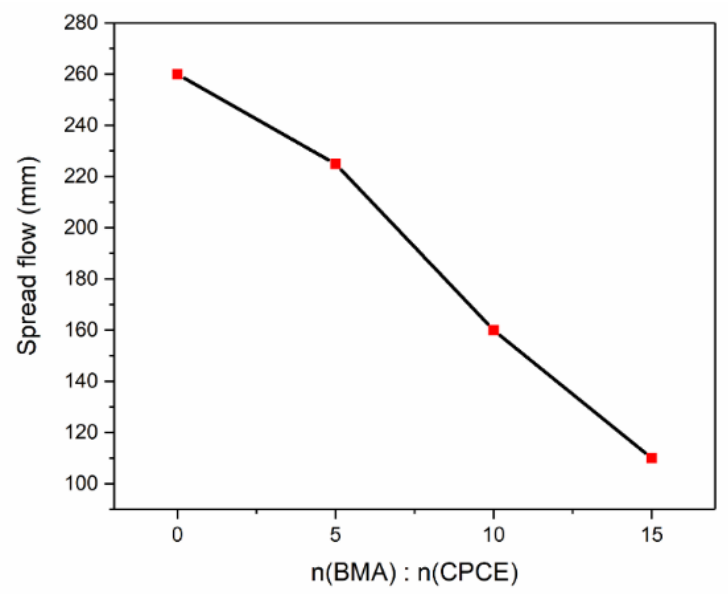

Fig. 7. Effect of BMA on the dispersing ability of SPCE

As revealed in Fig.6, with the BMA increased from 0 to 15 (molar ratio with CPCE), the peak belongs to the arm CPCE became smaller, which demonstrates that a much more yeilds of SPCE were obtained. However, more BMA led to larger molecular weight, so when the cement paste was performed to investigate the effect of BMA, a gradual decrease of the flow was observed (Fig.7). As the BMA reached 15, the paste lost its fluidity. So the molar ratio of BMA to CPCE was set as 5:1. The final molar ratio in synthesizing SPCE was then determined as CPCE: EGD: $\mathrm{BMA}=1: 3: 5$.

\section{Conclusions}

In summary, this paper offers a novel approach for constructing PCE with more complicated topological structure. A core-crosslinked star PCE (SPCE) was obtained by crosslinking the comb PCE (CPCE) with introduction of EGD and BMA. The structure of SPCE was clearly characterized by 1HNMR, FT-IR and GPC. Cement paste dispersing experiments illustrated that higher crosslinking density led to smaller spread flow. The introduction of spacing monomers promoted the formation of star structure, however, decreased the dispersing ability. The final synthetic procedure of SPCE was determined as CPCE: EGD: $B M A=1: 3: 5$. Further researches, such as improving the conversion yield of SPCE or exploring the effect of SPCE on the cement hydration, would be performed to investigate the dispersing mechanism of SPCE.

\section{Acknowledgements}

This work was financially supported by the China Postdoctoral Science Foundation (No. 2019M662898), Guangzhou Municipal Construction Co., Ltd. and Guangzhou Institute of Building Science Co., Ltd. (Nos. 2019-KJ020 and 2019Y-KJ09).

\section{References}

1. J. Liu, C. Yu, X. Shu, Q. Ran and Y. Yang, Cement Concrete Res., 124, 105834 (2019).

2. J. Plank, E. Sakai, C. W. Miao, C. Yu and J. X. Hong, Cement Concrete Res., 78, 81-99 (2015).

3. X. Liu, J. Guan, G. Lai, Z. Wang, J. Zhu, S. Cui, M. Lan and H. Li, J. Colloid Interf. Sci., 504, 12-24 (2017).

4. S. Sha, M. Wang, C. Shi and Y. Xiao, Constr. Build. Mater., 233, 117257 (2020).

5. S. Chen, S. Sun, X. Chen, K. Zhong, Q. Shao, H. Xu and J. Wei, Colloids and Surfaces A: Physicochemical and Engineering Aspects, 590, 124464 (2020).

6. D. Xie, J. G. Park and J. Zhao, Dent. Mater., 23, 395-403 (2007).

7. D. Xie, J. G. Park and J. Zhao, Dent. Mater., 23, 395-403 (2007).

8. Y. Zhao, F. Nian, H. Pang, J. Huang, H. Zhao, K. Wang and B. Liao, J. Appl. Polym. Sci., 46312 (2018).

9. C. Zhang, M. Miao, X. Cao and Z. An, Polym. Chem.-UK, 3, 2656-2664 (2012).

10. X. Shi, M. Miao and Z. An, Polym. Chem.-UK, 4, 1950-1959 (2013). 